A N N A L E S Annales de Bretagne et des Pays de l'Ouest

Anjou. Maine. Poitou-Charente. Touraine

110-4 | 2003

Espace et histoire

\title{
Ce que peut dire la géographie
}

L'exemple de l'organisation de l'espace minier dans le bassin houiller du Nord-Pas-de-Calais, approche théorique

\section{Guy Baudelle}

\section{(2) OpenEdition}

\section{Journals}

Édition électronique

URL : http://journals.openedition.org/abpo/1332

DOI : $10.4000 /$ abpo.1332

ISBN : 978-2-7535-1492-8

ISSN : 2108-6443

Éditeur

Presses universitaires de Rennes

Édition imprimée

Date de publication : 20 décembre 2003

Pagination : $55-66$

ISBN : 978-2-86847-933-4

ISSN : 0399-0826

\section{Référence électronique}

Guy Baudelle, "Ce que peut dire la géographie », Annales de Bretagne et des Pays de l'Ouest [En ligne],

110-4 | 2003, mis en ligne le 20 décembre 2005, consulté le 30 avril 2019. URL : http:// journals.openedition.org/abpo/1332 ; DOI : 10.4000/abpo.1332 


\title{
Ce que peut dire la géographie L'exemple de l'organisation de l'espace minier dans le bassin houiller du Nord-Pas-de-Calais. Approche théorique
}

\author{
Guy BAUDELLE \\ Professeur de Géographie de l'aménagement \\ Université Rennes 2 Haute-Bretagne
}

Nous voudrions simplement montrer ici la façon dont la géographie peut éclairer l'organisation d'un espace, appréhendé en l'occurrence sur la longue durée, puisque nous avons étudié l'exploitation du bassin houiller du Nord et du Pas-de-Calais (France) sur toute sa durée, c'est-à-dire de 1720 à 1990 , soit plus de deux siècles et demi ${ }^{1}$. En se permettant ainsi de transgresser les découpages entre périodes, le géographe fait sans doute preuve d'une première différence avec l'historien, pareille audace manifestant peutêtre son inculture historique...

Nous avons cherché à saisir les logiques de l'organisation de l'espace géographique par l'exploitation souterraine, à comprendre, en somme, la production de l'espace minier, pour reprendre l'expression d'Henri Lefebvre, particulièrement bienvenue ici ${ }^{2}$. Nous sommes partis pour cela d'une double interrogation initiale : d'abord mettre en évidence le rôle de l'organisation d'un espace adéquat dans le fonctionnement de l'exploitation charbonnière, autrement dit procéder à une réévaluation de l'instance spatiale dans la construction d'un système de production; mais on voulait aussi tenter d'expliquer la conception et le fonctionnement de l'espace organisé par l'exploitant minier, dans la mesure où cette compréhension est indispensable pour éclairer la configuration actuelle d'un territoire profondément marqué par l'activité extractive. Si l'on procède à un retour sur le passé, c'est donc pour mieux éclairer l'organisation spatiale héritée de cette longue période d'exploitation. Il s'agit bien d'une géographie historique largement mise au service de la compréhension du présent et dans laquelle

1. BAUDELLE, Guy, Le Système spatial de la mine. L'exemple du bassin houiller du NordPas-de-Calais, Lille, Atelier national de reproduction des thèses, 2 tomes, 1228 p. (Thèse d'État, 1994, Université de Paris I).

2. LeFEBVRE, Henri, La Production de l'espace, Paris, Anthropos, 1974 et 1986, 485 p. 
une place essentielle est faite à la compréhension de l'espace stricto sensu, à " l'espace pour lui-même " selon l'expression des organisateurs du colloque, sans pour autant négliger la société qui l'engendre et y évolue. Un tel projet a d'autant plus d'intérêt que la " nouvelle géographie », qui s'est épanouie dans les années 1960-1970 à la recherche de lois générales de l'espace, a souligné les lacunes de l'explication de l'organisation interne des bassins miniers ${ }^{3}$.

Figure 1 - L'espace, outil de reproduction du système d'exploitation minière (Sources : DAUPHINÉ, André, Les modèles de simulation en géographie, Paris, PUF, 1981.)

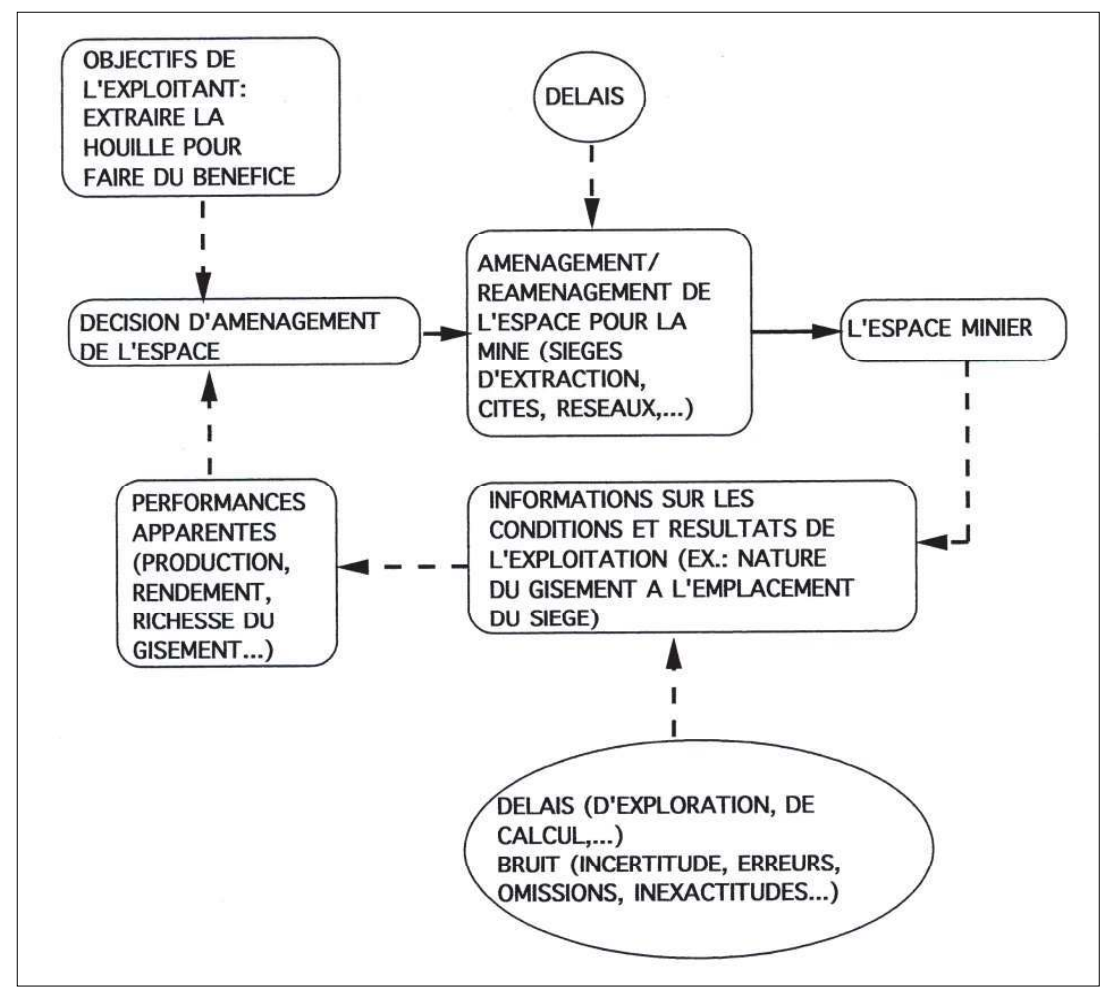

On se contentera ici de montrer comment l'utilisation d'un type de modèle théorique, relatif à la diffusion spatio-temporelle, permet de rendre compte du déroulement de l'expansion spatiale de la mise en valeur du gisement.

Rappelons en guise d'introduction que la communauté des géographes admet aujourd'hui le principe d'une interaction entre espace et société :

3. ABLER, Ronald, AdAMS, John et Gould, Peter, Spatial organization. The geographers view of the world, Londres, Englewood Cliffs, Prentice Hall, 1971, 587 p. 
l'espace n'est pas seulement le produit de la société, il agit aussi en retour sur elle, ce qui fait système. L'espace minier n'échappe pas à la règle : la construction spatiale est autant la cause que la conséquence de l'exploitation minière, si bien que l'espace est une sortie du système d'exploitation mais aussi une entrée, formant une boucle capitale (figure 1).

Figure 2 - L'extension du champ d'exploitation, principal remède à la raréfaction des réserves (Source : DAUPHINÉ, André, Les modèles de simulation en géographie, Paris, PUF, 1981.)

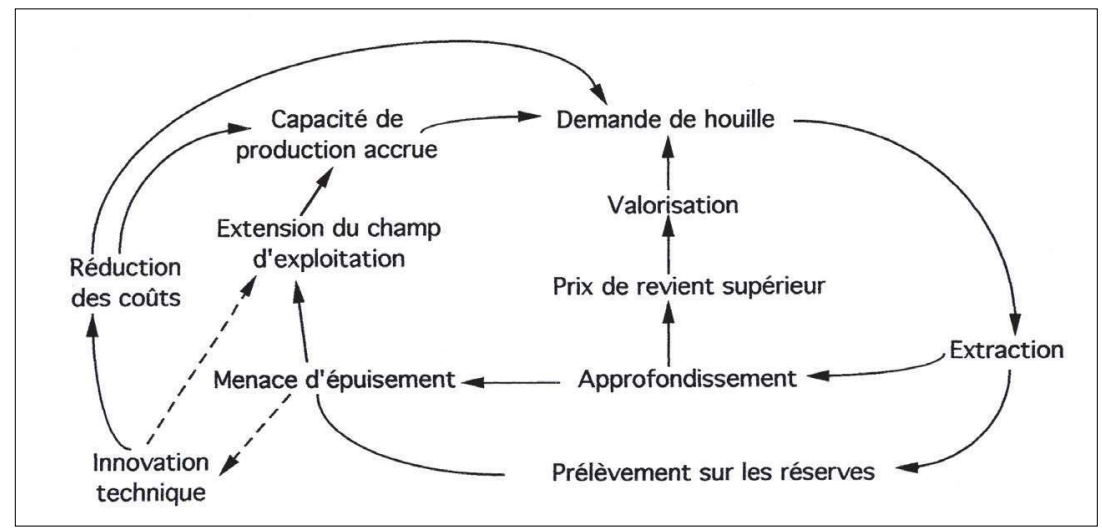

Dans le cas de l'exploitation minière, une réorganisation permanente de l'espace est nécessaire pour maintenir ou augmenter la production : l'aménagement de l'espace en surface et au fond permet d'accroître la production, mais ce prélèvement même oblige sans cesse à poursuivre les aménagements pour maintenir cette production. Pour conserver des réserves, on doit sans cesse reconnaître de nouveaux gisements exploitables : c'est une course perpétuelle, sous peine de mourir d'inanition comme le dit, en 1837, l'appel de fonds lancé aux directeurs de la Compagnie des mines d'Aniche en vue d'ouvrir une nouvelle fosse destinée à maintenir le tonnage ${ }^{4}$. Le meilleur moyen réside dans l'extension du champ d'exploitation (figure 2) à cause de la loi du rendement décroissant qui s'applique aux travaux souterrains, comme le résumait le même document de la Compagnie d'Aniche : " plus nos travaux avancent, plus l'extraction devient coûteuse ". On a donc plus intérêt à déployer l'exploitation dans l'espace qu'à l'approfondir, la pente des coûts étant plus faible horizontalement que verticalement.

S'est ainsi déployée une véritable " conquête spatiale ", pour prolonger la métaphore de Marcel Gillet sur " la conquête du bassin du Nord ${ }^{5}$ ", véri-

4. Assemblée des directeurs de la Compagnie, 1810-1837, Arch. dép. du Nord, J 701/16.

5. GiLlet, Marcel, Les Charbonnages du Nord de la France au $19^{e}$ siècle, Paris, La Haye, Mouton, 1973, $527 \mathrm{p}$. 
table « colonisation minière ${ }^{6}$ " ayant donné lieu à la spectaculaire occupation d'un espace où les hommes se sont accumulés en un long sillon comptant aujourd'hui un million d'habitants, soit plus de $600 \mathrm{au} \mathrm{km}{ }^{2}$, autorisant des comparaisons avec la mise en valeur des pays neufs.

Il s'agit de rendre compte d'abord de la progression de l'exploration du bassin, puis du déroulement de sa mise en valeur.

\section{Expliquer la progression de la découverte du gisement}

La carte 1 montre les progrès de l'identification des contours du gisement, qui forme une bande de $120 \mathrm{~km}$ sur 15. L'avancée de la reconnaissance de ces limites a constitué une "frontière " permanente, ce que des auteurs anglo-saxons appellent la resource frontier, front de repérage qui a fait de l'ingénieur des mines une sorte de pionnier. La date de la découverte - et donc de la mise en valeur, qui la suit généralement de peu - ayant fortement influencé la configuration actuelle des lieux compte tenu des techniques et des besoins propres à chaque période, reconstituer la chronologie de l'exploration puis l'expliquer constitue une étape essentielle pour la compréhension de l'organisation spatiale actuelle en surface.

\section{Carte 1 - La progression de la reconnaissance de l'extension du gisement}

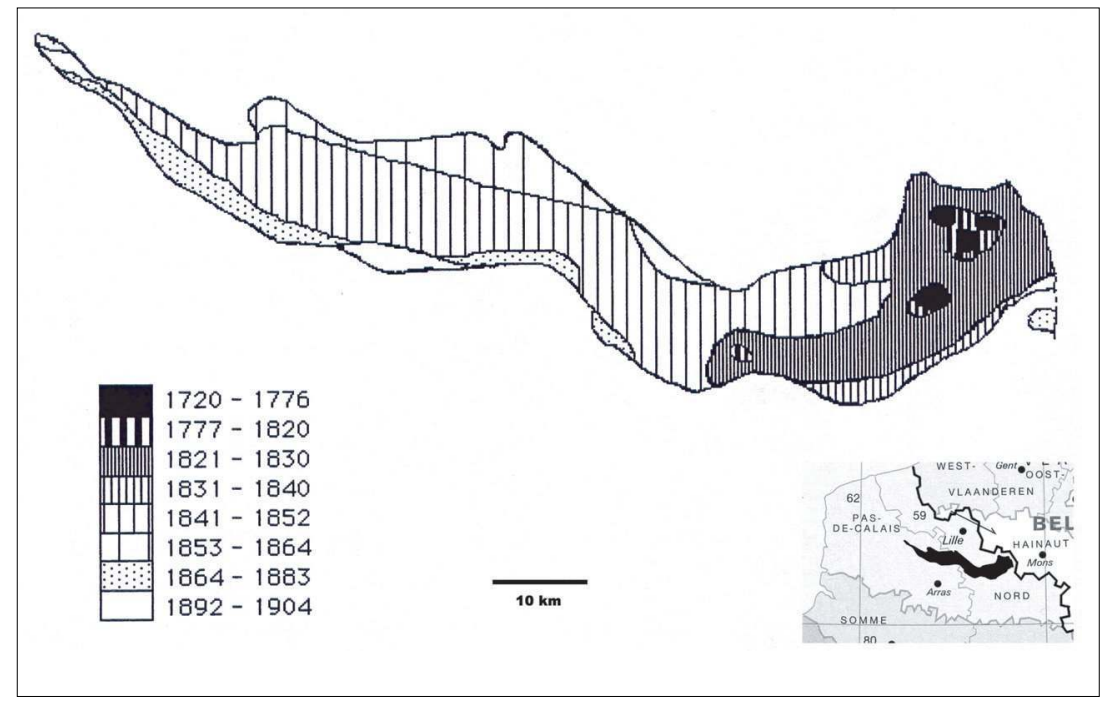

La carte peut s'interpréter à l'aide des modèles de diffusion spatio-temporelle introduits par la nouvelle géographie dont ils constituent même les

6. SMAILES, Arthur E., North England, Londres, Thomas Nelson and S., 1960, 324 p. 
travaux fondateurs, nés en Suède dans les années $1930^{7}$. Ces modèles s'attachent à comprendre la diffusion d'une innovation, terme désignant tout phénomène nouveau apparaissant en un point de l'espace. Le précurseur Torsten Hägerstrand étudia ainsi la diffusion géographique de la TSF dans les foyers suédois. Ici, l'innovation est constituée par la découverte du charbon (puis sa mise en valeur). Plusieurs types de diffusion ont été identifiés (figure 3). On va voir que cette mobilisation théorique apporte une réelle valeur ajoutée scientifique à la compréhension du phénomène étudié.

On s'aperçoit d'abord que, globalement, la progression de l'exploration s'est effectuée par contiguïté, selon une diffusion dite aussi " par contagion ". Un tel processus n'est pas le fait du hasard, mais reflète le raisonnement des premiers ingénieurs des mines eux-mêmes qui ont longtemps cherché la précieuse houille dans le prolongement des veines existantes, déterminant de la sorte une avancée de l'Est - où a été découvert le gisement français près de la nouvelle frontière décidée au traité d'Utrecht (1713) - vers l'ouest, d'autant que la profondeur croissante des couches d'est en ouest a retardé la découverte de la houille vers le couchant.

Secondairement, les ingénieurs ont obéi à une conception linéaire de la géologie minière : induits en erreur par le gisement de l'actuel Hainaut belge de l'autre côté de la frontière, disposé est-ouest et exploité depuis le Moyen Âge, les exploitants ont longtemps cru à une orientation longitudinale (est-ouest) systématique des veines de charbon, d'autant que les premiers ingénieurs étaient souvent wallons, et même à une profondeur constante des couches. La foi... du charbonnier en ces pseudo-lois géologiques explique en partie la découverte tardive de la partie occidentale du bassin français, en raison de l'inflexion des couches vers le nord-ouest dans sa partie centrale (carte 1). Le gisement du Pas-de-Calais n'a été découvert qu'en 1847, après de vaines recherches vers Arras. En termes théoriques,

7. HäGERSTRAND, Torsten, The Propagation of innovation waves, Lund, Lund Studies in Geography, Série B, n 4 , 1952, 21 p.
Figure 3 - Les types de diffusions

(Source : DAUPHINÉ, André, Les

modèles de simulation en géographie, Paris, PUF, 1981.)

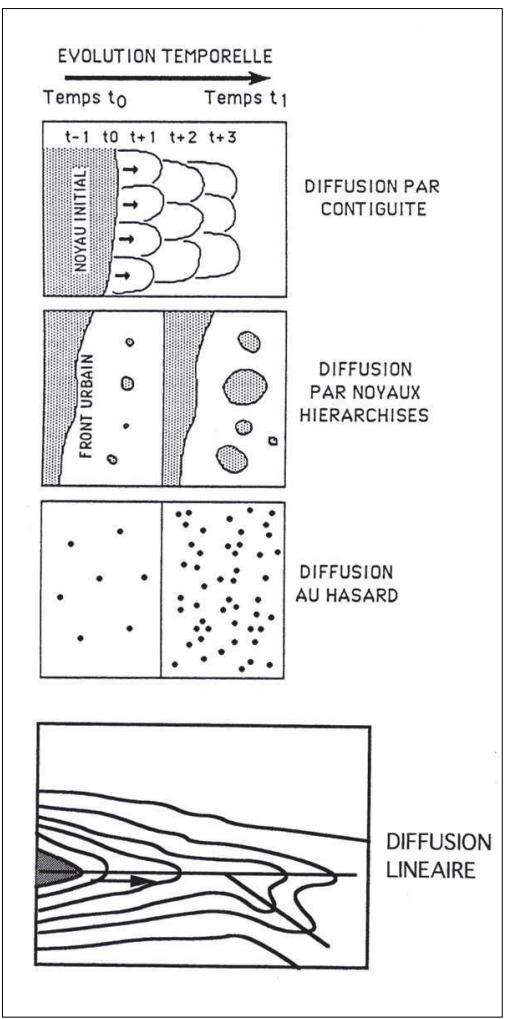


on dira que les ingénieurs supposaient une autocorrélation spatiale supérieure à ce qu'elle est en réalité. Le concept d'autocorrélation spatiale repose sur l'hypothèse qu'un lieu ressemble plus aux lieux voisins qu'à des points plus éloignés. Il fait référence à la probabilité de trouver la même chose en B que dans l'espace voisin A. L'autocorrélation spatiale exprime donc l'intensité de la relation entre la proximité des lieux et leur degré de ressemblance.

À moyenne échelle, on retrouve cette logique de diffusion linéaire (figure 3), en raison du rôle des voies de circulation (fluviales, navigables et routières) dans l'organisation du repérage, les facilités de communication permettant l'installation plus commode du matériel de forage et laissant espérer de meilleures conditions de transport ultérieur des produits. Les chances de découverte étaient donc logiquement supérieures à proximité des voies d'eau, en particulier l'Escaut. Notons que cette interférence entre modalités de la mise en valeur et faits de surface est une constante de l'histoire de l'exploitation du bassin, alors que la littérature a souvent estimé que les considérations relatives au sous-sol l'emportaient sur la prise en compte de l'organisation de l'espace, pourtant d'une appréhension beaucoup plus immédiate pour les exploitants que la configuration du gisement proprement $\mathrm{dit}^{8}$.

Dans le détail, la reconnaissance s'est partiellement effectuée par noyaux hiérarchisés (figure 3 ) autour des puits d'importance variable : l'exploration est d'autant plus rapide que les fosses sont bien équipées et demandent à être " saturées ", c'est-à-dire alimentées. Jusqu'aux années 1950, les différents sièges d'extraction ne connaîtront toutefois qu'une faible différence respective de puissance et de rayon d'action.

Dans le temps, la progression n'a en revanche pas suivi un cours linéaire, mais irrégulier en raison de l'intervention d'éléments aléatoires. En règle générale, le rythme de diffusion d'une innovation dans le temps dessine une courbe dite logistique (en forme de $S$ écrasé) (figure 4). Cela se produit quand l'effet de voisinage est homogène, ce dernier ne produisant paradoxalement pas une vitesse de diffusion constante, mais au contraire différentes phases d'adoption plus ou moins rapide. La confrontation de notre cas au modèle permet de reconnaître l'allure grossièrement logistique de la courbe de diffusion : lenteur initiale par méfiance ou méconnaissance; croissance rapide ensuite par mimétisme au cours de la phase d'expansion; rendement décroissant de la phase finale de saturation. Cela permet aussi de repérer les écarts au modèle et de faire apparaître les effets inhibants ou sollicitants d'événements ayant ralenti ou accéléré le repérage du gisement par rapport aux modalités ordinaires d'une diffusion. On constate que le démarrage a été ici particulièrement lent, avec en revanche une phase d'expansion en conséquence plus brève et spectaculaire cor-

8. BAUdELle, Guy, " La carte, outil de réflexion de l'ingénieur des mines ", Mappemonde, 1990, n 2, p. 2-5; " Modèles spatiaux, modèles géonomiques et contraintes naturelles ", Groupe Dupont, Géopoint 1996, Avignon, Université d'Avignon, 1997, p. 33-35. 
Figure 4 - Le rythme de repérage des contours du gisement : une courbe grossièrement logistique

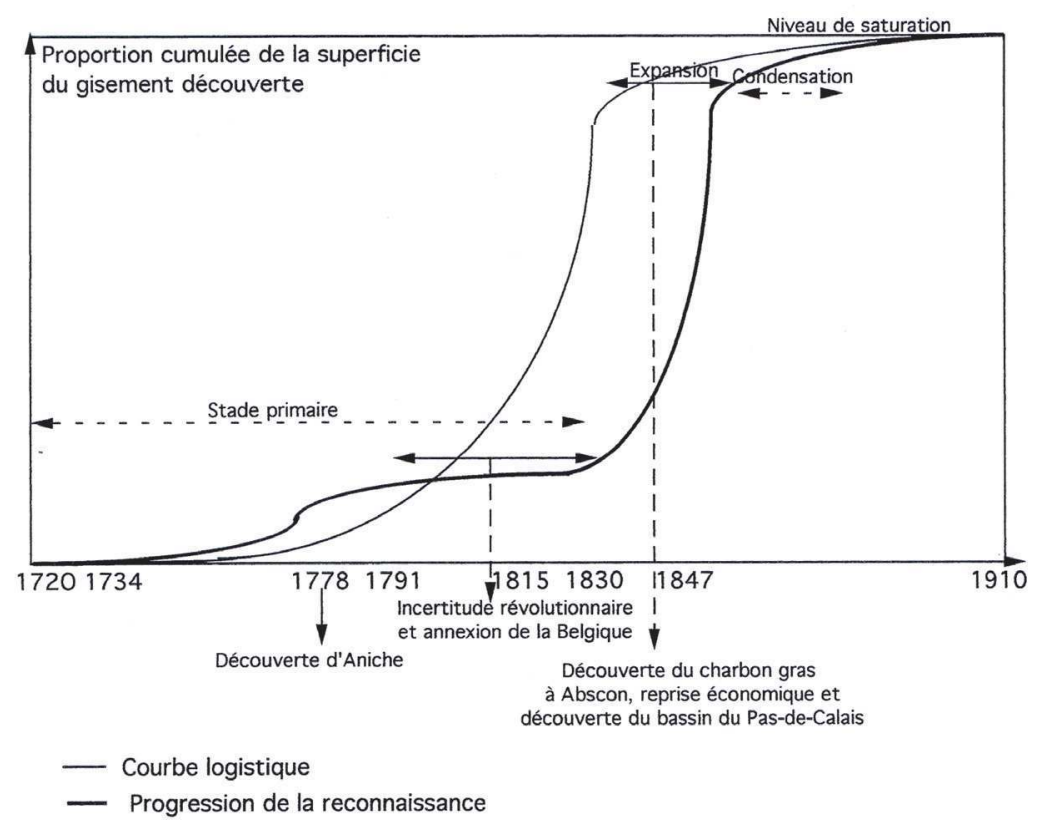

respondant à la reconnaissance et à la mise en valeur du Pas-de-Calais, période que les Houillères du Bassin du Nord et du Pas-de-Calais ellesmêmes ont appelé " la conquête de l'Ouest ".

Le modèle présente le même intérêt explicatif pour la progression de la mise en exploitation.

\section{Expliquer les progrès de la mise en valeur}

En s'appuyant sur la figure 2, on peut montrer que le principe de la propagation d'une onde d'innovation peut rendre compte de la mise en œuvre de l'exploitation proprement dite. La figure 3, qui schématise la précédente, révèle que le schéma général de mise en valeur répond à une même logique de contiguïté : on retrouve globalement une onde principale d'est en ouest. Cette diffusion par voisinage se vérifie aussi à moyenne échelle : on voit bien (carte 2) comment les points connus par où a débuté l'exploitation, figurés en noir (Valenciennes-Anzin, Fresnes et Vieux-Condé au nord-est, puis Aniche et Denain), ont fonctionné comme des centres de diffusion, c'est-à-dire de progression de l'exploitation. Enfin, l'observation est également valable à grande échelle : l'extraction autour de chaque puits réduit progressivement, de proche en proche, les aires inexploitées.

Ce mode de diffusion, dit aussi par expansion, diffère de la diffusion dite par migration qui en constitue une variante. Cette autre modalité se rencontre 
dans des gisements exploités par tranches d'épuisement successif avec abandon quasi total d'une partie ancienne - la ceinture interne des auteurs anglais comme L. D. Stamp ${ }^{9}-$, puis déplacement complet de l'exploitation par création d'un nouveau front dans une zone encore vierge, la ceinture externe faisant figure de gisement neuf, comme dans le Nord-Est anglais voire dans la Ruhr. Un tel cycle spatial est analogue aux brutaux fronts d'exploitation agricole des plantations coloniales avec épuisement des sols.

Carte 2 - La progression de la mise en exploitation ou le front de la colonisation minière : période de mise en service des sièges

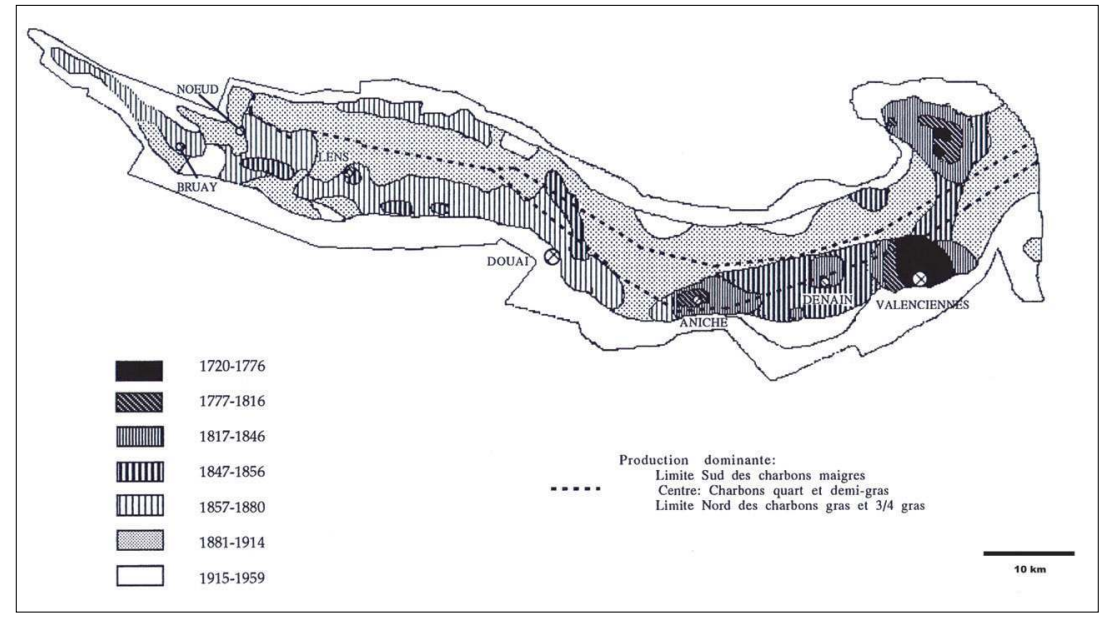

Dans la partie sud du gisement, l'extraction du charbon a par ailleurs obéi à une logique de propagation linéaire, axiale, en raison d'un stimulant exceptionnel : la présence de charbon cokéfiable, le plus demandé, dans toute la partie sud du bassin. La mise en valeur du charbon à coke pourtant plus profond a ainsi précédé l'exploitation d'autres variétés plus proches de la surface mais moins demandées. Ainsi s'expliquent l'exploitation plus précoce du faisceau méridional (carte 3) et les regrets de la Compagnie des mines d'Anzin déplorant en 1892 que cette variété grasse représente $30 \%$ de sa production et $10 \%$ de ses ressources : «malheureusement [...] il faut bien produire uniquement ce que l'on peut vendre. Les générations futures sauront peut-être mieux apprécier le charbon maigre [...] que la Compagnie d'Anzin peut produire à très bas prix et en grande quantité ${ }^{10}$ ". L'effet de corridor en résultant est incontestable (carte 2). Inversement, l'exploitation plus tardive des couches septentrionales des-

9. STAMP, Lawrence D., The British Isles. A geographic and economic survey, Londres, Longmans, Green \& Cy, 1937, 719 p.

10. Rapport à la Régie de la Compagnie des Mines d'Anzin, $1^{\text {er }}$ sem. 1892, Centre Historique Minier de Lewarde (Nord), 11 W 6. 
sine une onde secondaire (carte 3). Comme pour la découverte, cette modalité linéaire a été renforcée par l'existence de voies de transport; l'Escaut notamment a d'autant plus facilité l'exploitation qu'il coule sur le charbon gras! Le chemin de fer, ensuite, a eu le même effet sur le... drainage de la production.

Carte 3 - Onde principale, vague secondaire :

\section{modèle spatio-temporel de la progression de la mise en exploitation} du bassin du Nord-Pas-de-Calais

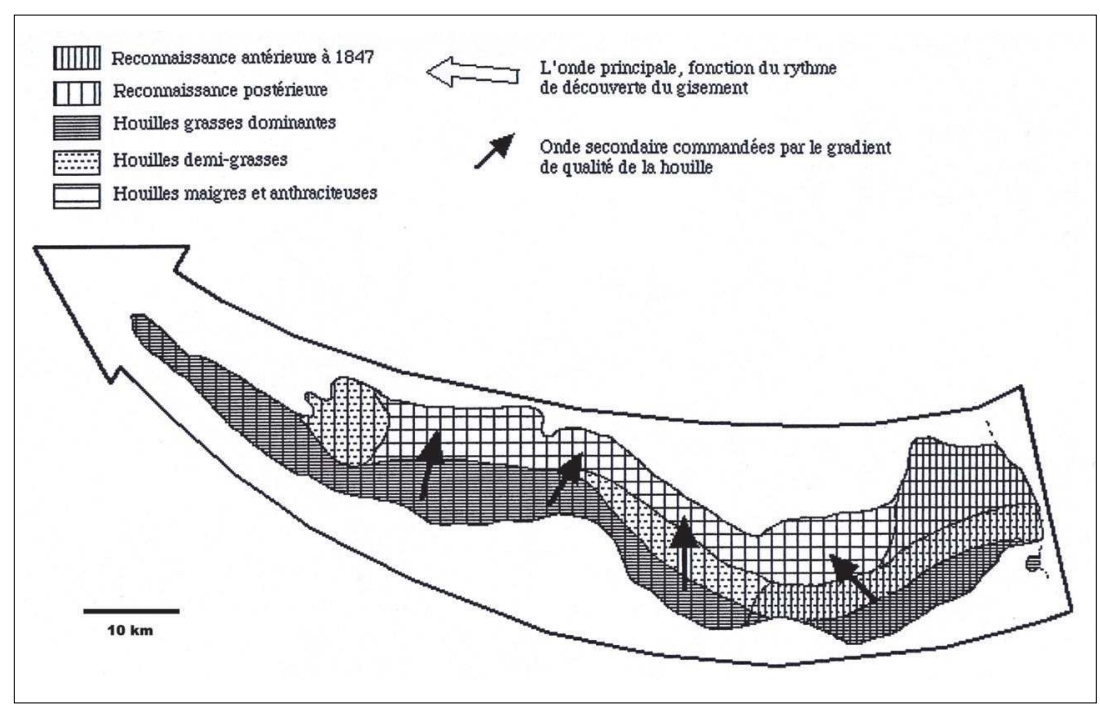

À grande échelle enfin, localement, deux autres facteurs expliquent les résidus, c'est-à-dire les écarts au modèle général de mise en valeur par contiguïté. Des difficultés techniques d'abord, en particulier la présence de terrains aquifères, difficiles à franchir avant l'invention de la technique de la congélation à l'extrême fin du $19^{\mathrm{e}}$ siècle. La carte 4 montre par exemple l'interruption des couches exploitées à l'est de Denain, à l'emplacement du fameux torrent d'Anzin, celui-là même qui a inspiré Zola en ces termes dans Germinal : "Cette mer souterraine, terreur des houillères du Nord [...] roulant ses flots noirs à plus de 300 mètres du soleil. " L'autre cause de déviation par rapport au modèle a été la dimension des concessions. Toutes choses égales par ailleurs, la vitesse de propagation des sièges d'extraction à l'intérieur d'une concession fut en effet inversement proportionnelle à sa superficie, comme on le constate aisément sur la carte 4 : la minuscule Compagnie de Vicoigne exploite ses seules ressources alors qu'autour la grande Compagnie d'Anzin pourvue d'un immense territoire peut bien négliger ces mêmes faisceaux de charbon maigre. 
Carte 4 - Solutions de continuité dans la mise en valeur du gisement (Source : A. Burat, Les Houillères de France en 1866, Paris, Libr. Polytechn. J.Baudry, 1867.)

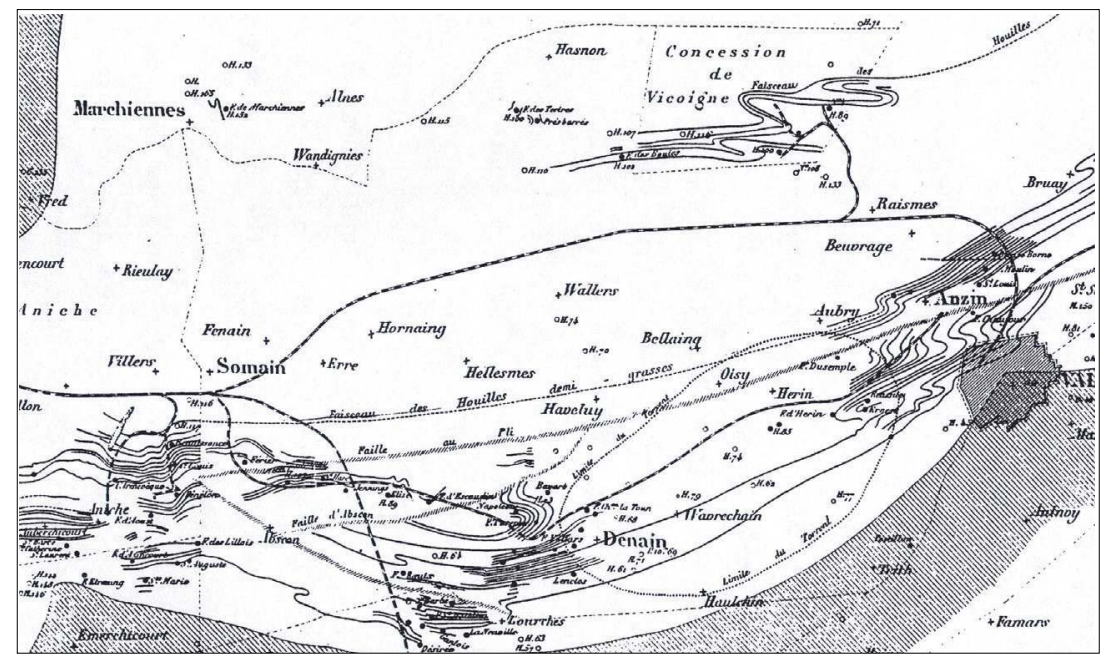

Enfin, on peut de la même façon étudier le rythme du démarrage de la mise en valeur du gisement, qui suit aussi globalement le modèle de la courbe logistique observé pour la découverte. On peut mesurer l'avance ou le retard de la mise en exploitation par rapport au rythme non pas théorique compte tenu du modèle, mais par rapport à la vitesse moyenne de la diffusion effective de l'exploitation. Pointer les écarts positifs ou négatifs par rapport au rythme d'avancée général de la propagation de l'extraction permet en effet de mesurer les effets-barrières (facteurs de retard) ou les effets d'accélération et de localiser les endroits où l'exploitation a stagné comme ceux où elle s'est accélérée au contraire tout en datant ces points d'inflexion. Pour cela, on recourt à la technique de la surface de tendance (carte 5). Pour dresser une telle carte, on a cherché à évaluer la relation entre la date de mise en exploitation et la distance à la frontière belge (où a commencé l'exploitation) en procédant à une analyse statistique de régression, au résultat honorable avec un coefficient de corrélation assez élevé $(0,69)$. Compte tenu de la faible largeur du bassin, on s'est contenté de découper le bassin selon un transect longitudinal d'est en ouest par sections de $5 \mathrm{~km}$. On a ensuite cartographié la surface de tendance linéaire indiquant la date de mise en valeur donnée par la régression. On peut alors confronter la date effective de mise en valeur avec l'année-tendance donnée par la régression. On constate que les écarts principaux s'expliquent essentiellement par des différences de capacités techniques : les forts résidus négatifs (qui expriment un retard relatif de la mise en valeur par rapport au rythme général) se rencontrent localement dans les secteurs aquifères de l'Est, à proximité de forts résidus positifs correspondant aux 
exploitations de la Compagnie des mines d'Anzin, la première constituée; d'autres résidus négatifs s'observent plus généralement dans le Pas-deCalais, c'est-à-dire dans la moitié occidentale, sauf à l'extrême ouest puisque, une fois découverte, la mise en exploitation de cette partie du gisement a été très rapide.

Carte 5 - Surface de tendance linéaire est-ouest de la mise en exploitation du bassin houiller du Nord-Pas-de-Calais

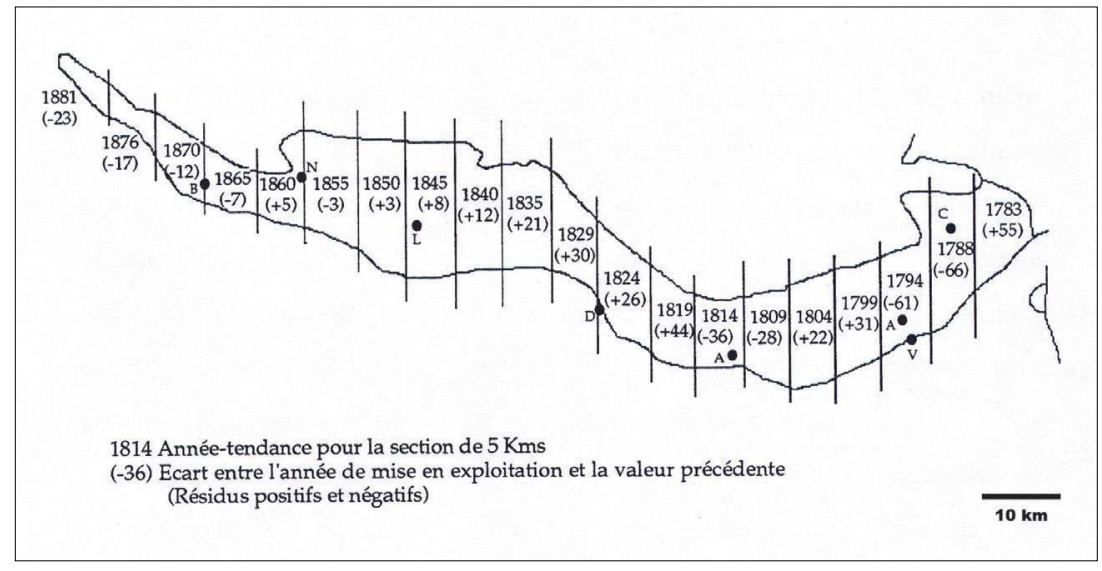

Bien sûr, si l'on voulait rendre compte ensuite de l'inégale intensité de l'exploitation, autrement dit du rythme plus ou moins rapide du prélèvement de la ressource, il faudrait faire intervenir bien d'autres éléments, au premier rang desquels le marché et le prix de revient, ce qu'on ne développera pas ici (faute d'espace!).

Pour finir, ajoutons que les modèles de diffusion spatio-temporelle peuvent naturellement s'appliquer aussi au recul de l'exploitation, à son reflux : "l'innovation " dont on étudie la propagation est alors la fermeture des puits. Une carte générale simplifiée n'est plus forcément alors la meilleure représentation, car la contraction de l'exploitation opère cette fois par noyaux hiérarchisés (figure 3) selon une diffusion hiérarchique ascendante, des plus petits sièges d'extraction vers les plus grands : c'est l'inverse de la diffusion la plus courante des innovations technologiques ou sociales, qui se propagent des plus grandes villes vers les centres de peuplement les plus petits. Il est assez logique d'observer une modalité inversée ici car il s'agit d'un phénomène d'abandon plutôt comparable au processus de dépeuplement des régions rurales ou des espaces situés aux marges de l'œkoumène par exemple.

En conclusion, soulignons l'intérêt des modèles de diffusion pour montrer l'ordre caché de l'espace minier : le recours à de tels modèles permet 
de révéler ce qu'Henri Reymond appelle une géographie clandestine ${ }^{11}$ insoupçonnée autrement, celle d'une économie souterraine dictant les phases de mise en valeur et, in fine, l'organisation actuelle de l'espace en surface, qui reste l'objet central du géographe ${ }^{12}$.

\section{RÉSUMÉ}

À partir de l'exemple du bassin houiller du Nord et du Pas-de-Calais (France), mis en valeur de 1720 à 1990, on montre comment l'utilisation de modèles géographiques théorisant la diffusion spatio-temporelle d'un phénomène permet de rendre compte du déroulement spatial de la mise en valeur de cette ressource. On montre ainsi que la découverte du gisement comme son exploitation ont obéi à une logique de diffusion temporelle conforme à la règle générale. Dans l'espace, cette progression à également obéi à différentes modalités de diffusion décrites par les modèles. La mise en évidence des écarts au modèle (dits résidus) permet de mettre en évidence les variations locales du cheminement de l'exploitation et de les expliquer. Ces modèles de diffusion peuvent également s'appliquer au repli de l'exploitation.

\section{ABSTRACT}

From the example of the Nord and Pas-de-Calais coalfield (France), active from 1720 to 1990, one shows how the use of geographical models theorizing the spacetime diffusion of a phenomenon makes it possible to account for the resource exploitation space course. First we show that the field discovery and its exploitation as well obeyed a time diffusion logic in conformity with the general rule. In space, this progression also obeyed various diffusion processes described by the models. Showing the deviations to the model (known as residuals) allows to highlight the local variations of the exploitation course and to explain them. These diffusion models can also be applied to the exploitation recession.

11. REYMOND, Henri, « Une problématique théorique de la géographie : plaidoyer pour une chorotaxie expérimentale ", dans ISNARD, H., RACINE, J.-B. et ReYmond, H., Problématiques de la géographie, Paris, PUF, 1981, p. 163-262.

12. Bibliographie complémentaire : DAUPHINE, André, Les Modèles de simulation en géographie, Paris, Economica, 1987, 187 p.; SAINT-Julien, Thérèse, La diffusion spatiale des innovations, Montpellier, Reclus, 1985, 40 p. 